\title{
Resistencia a antibióticos $\beta$-lactámicos y eritromicina en bacterias de la cavidad oral
}

\author{
Resistance to $\beta$-lactam Antibiotics and Erythromycin \\ in Bacteria of the Oral Cavity
}

González Norma Enid ${ }^{1}$, Zapata Alejandro Cuartas ${ }^{2}$, Sánchez-Henao Diego Fernando ${ }^{3}$, Chávez-Vivas Mónica ${ }^{4}$

\section{Resumen}

Introducción. La microbiota humana como fuente de bacterias y genes de resistencia constituyen un problema de salud pública. En este estudio se investigó la prevalencia de bacilos entéricos Gram negativos resistentes a $\beta$-lactámicos y de los Streptococcus del grupo viridans (EGV) con resistencia a eritromicina en la cavidad oral. Métodos. Se realizó un estudio descriptivo de corte transversal con 193 aislamientos de la cavidad oral sana de 178 adultos que asistieron a una Clínica Odontológica de la ciudad de Cali durante el 2018. La evaluación de la sensibilidad antimicrobiana se realizó en 59 bacilos entéricos y 134 EGV y se identificó por PCR los genes que confieren resistencia a $\beta$-lactámicos y eritromicina. El análisis estadístico se realizó mediante el empleo del paquete SPSS vs 23. Resultados. El $84,7 \%$ de los bacilos entéricos fueron multirresistentes y presentaron genes bla, siendo blaTEM-1 $(49,2 \%)$ y blaVIM-2 $(30,5 \%$,$) los más prevalentes. Los EGV fueron resistentes$ a eritromicina $(38,8 \%)$ y clindamicina $(28,4 \%)$. El $18,7 \%$ presentaron el fenotipo cMLS $\beta$, $4,5 \%$ el iMLS $\beta$ y el $14,9 \%$ fueron M. El gen ermB se detectó en los cMLS $\beta$, $(13,4 \%)$ y el gen mef en los M (9,7\%). Conclusión. En este estudio se demostró la presencia de EGV y bacilos entéricos resistentes a los antibióticos y portadores de genes de resistencia a

\footnotetext{
1. Facultad de Ciencias de la Salud. Universidad Libre, Seccional Cali. Colombia.

Correo electrónico: normaegv@hotmail.com

ORCID: https://orcid.org/0000-0001-6896-3514

2. Programa de Medicina. Facultad Ciencias de las Salud. Universidad Libre, Seccional Cali. Colombia.

Correo electrónico: donalejandro88@hotmail.com

ORCID: https://orcid.org/0000-0003-3539-0531

3. Programa de Odontología. Facultad de Salud. Universidad Santiago de Cali. Colombia.

Correo electrónico: difesa1967@gmail.com

ORCID: https://orcid.org/0000-0001-6511-199X

4. Programa de Medicina. Facultad de Ciencias de la Salud. Grupo de investigación GIMMEIN. Universidad Libre, Seccional Cali. Colombia.

Correo electrónico: monikchavez@gmail.com

ORCID: https://orcid.org/0000-0001-9996-3744

Correspondencia: monikchavez@gmail.com
} 
eritromicina y genes bla en la cavidad oral sana. La presencia de estas bacterias representa un riesgo para la salud de los individuos portadores y contribuyen a la creciente epidemia de resistencia bacteriana.

Palabras claves: Enterobacteriaceae, bacilos entéricos Gram negativos, Streptococcus del grupo viridans, resistencia, antibióticos, microbiota oral, $\beta$-lactámicos, eritromicina.

\section{Abstract}

Introduction. The human microbiota as a source of bacteria and resistance genes is a public health problem. This study researched the prevalence of Gram-negative enteric bacilli resistant to $\beta$-lactams and erythromycin resistance in the oral cavity. Methods. A descriptive cross-sectional study was carried out with 193 isolates obtained from the oral cavity of 178 healthy adults who were treated at a Dental Clinic in the city of Cali during 2018. The evaluation of antimicrobial sensitivity was performed in 59 enteric bacilli and $134 \mathrm{EGV}$ and the genes that confer resistance to $\beta$-lactam and erythromycin were identified by PCR. Statistical analysis was performed using the SPSS statistical package vs. 25.0. Results. $84.7 \%$ of the enteric bacilli presented the MDR phenotype and all presented the bla genes, blaTEM-1 (49.2\%) and blaVIM-2 (30.5\%) being the most prevalent. EGVs were resistant to erythromycin (38.8\%) and clindamycin (28.4\%). $18.7 \%$ presented the cMLS $\beta$ phenotype, $4.5 \%$ the iMLS $\beta$ and $14.9 \%$ were $\mathrm{M}$. The erm B gene was detected more frequently in the cMLS $\beta,(13.4 \%)$ and the mef gene in the M (9.7\%). Conclusion. This study demonstrated the presence of antibiotics and Gram-negative enteric bacilli resistant to antibiotics and carriers of erythromycin resistance genes and bla genes, respectively in the healthy oral cavity. The presence of these bacteria represents a risk to the health of carrier individuals and contributes to the growing epidemic of bacterial resistance.

Keywords: Enterobacteriaceae, Gram Negative Enteric Bacilli, Viridans Streptococcus Group, Antibiotic Resistance, Oral Microbiota, $\beta$-lactamic, Erythromycin. 


\section{Introducción}

La resistencia bacteriana a antibióticos constituye un grave problema de salud pública que cada vez se hace más complejo de tratar. La problemática se agudiza aún más con la aparición de organismos ambientales y de la microbiota humana portadoras de resistencia (1-3). Entre las cepas de Streptococcus del grupo viridans (EGV) se ha detectado una prevalencia creciente de resistencia a macrólidos, $\beta$-lactámicos y clindamicina (4-6). Otro grupo importante de bacterias que fácilmente desarrollan resistencia a los antibióticos lo constituye el grupo de bacilos Gram negativos de la familia Enterobacteriaceae. Este grupo tiene capacidad de colonizar y proliferar en la cavidad bucal haciendo parte de la microflora transitoria $(2,3,7-9)$.

Numerosos estudios señalan que las bacterias de la microbiota oral permanente y transitoria adquieren genes de resistencia en gran medida debido a la transferencia intermicrobiana a partir de patógenos a la flora normal (10-12). La elevada exposición de los EGV y de los bacilos Gram negativos a la penicilina y otros antibióticos $\beta$-lactámicos ha producido las condiciones ideales para la adquisición de enzimas $\beta$-Lactamasas que alteran y confieren la resistencia a estos antibióticos y son codificadas por los genes bla $(11,13)$. La antibioterapia con antibióticos de amplio espectro, como la eritromicina, también ha ejercido una presión selectiva sobre las bacterias orales. La resistencia a este antibiótico está mediada por la alteración del sitio de unión de la eritromicina al ribosoma por metilación de una enzima codificada por el gen emr, que induce un cambio conformacional que impide la unión a su lugar de acción, tanto de los macrólidos como de las lincosamidas y estreptograminas B. Este patrón fenotípico se denomina resistencia MLSB. La expresión del gen erm puede ser constitutiva o inducible $(14,15)$. Otros mecanismos de resistencia a la eritormicina lo constituyen la inactivación del antibiótico por la adición de un grupo fosfato al hidroxilo de posición 2' del amino azúcar por la acción de las fosfotranferasas codificadas por los genes $m p h A$. También se reporta una resistencia mediada por bombas de eflujo codificadas por los genes mefA y mefE (16).

El propósito del presente estudio fue investigar la prevalencia de bacilos entéricos Gram negativos resistentes a los antibióticos $\beta$-lactámicos y de los EGV con resistencia a eritromicina, y determinar si los genes que confieren resistencia a estos antibióticos son detectados en la cavidad oral sana de pacientes que consultaron una clínica odontológica de la ciudad de Cali durante el año 2018.

\section{Métodos}

Se realizó un estudio descriptivo de corte transversal, por lo cual se tomaron muestras de saliva y de placa dental de 178 adultos (96 mujeres y 82 hombres) con un promedio de edad de $44+13.56$ ańos, que asistie- 
ron la Clínica Odontológica de la ciudad de Cali y sometidos a profilaxis oral. Se incluyeron pacientes que no habían recibido tratamiento con antibióticos en los últimos 3 meses y no sufrían de alguna enfermedad oral. Una muestra de saliva $(2 \mathrm{ml})$ se recolectó en un recipiente estéril y las muestras de la placa dental se tomaron de frotis con un hisopo estéril de alginato de calcio. En el caso de las muestras de placa subgingival se tomaron de cuatro sitios diferentes con puntas de papel estériles y se agruparon en $4 \mathrm{ml}$ de solución de Ringer y se mezclaron con la muestra de saliva.

\section{Métodos de Microbiología Clásica} Aislamiento de bacterias entéricas y de EGV e identificación

Las muestras se resuspendieron en caldo Todd-Hewitt (Oxoid) y se sembraron en cada medio. Para el aislamiento de bacterias entéricas las diluciones se sembraron en agar Mc Conkey (Oxoid, Hampshire, United Kingdom) por 24 horas a $37 \mathrm{oC}$. El estudio microscópico se basó por el método de Gram para establecer morfología y tinción bacteriana y la posterior identificación se realizó con pruebas bioquímicas empleando el sistema automatizado Vitek GNI+ (bioMeriex Vitek Inc., Hazelwood, MO).

Para aislar los EGV se sembraron las muestras en medio selectivo agar mitis salivarius y agar chocolate base tripticasa-soya y se incubaron en una atmósfera de $\mathrm{CO} 2$ durante 2 días a $37^{\circ} \mathrm{C}$. Se seleccionaron las bacterias por su morfología celular con coloración de Gram y la identificación bioquímica se realizó por el sistema Api 20 Strep (Biomerieux). La confirmación de las cepas del grupo viridans se realizó por el método de dilución en caldo por Microscan System (Becton Dickison), la cual emplea un panel de 27 pozos para la identificación bioquímica con pruebas como pruebas cristal violeta, nitritos, Novobiacina, PGR, B-D Glucoronidasa, Indol Fosfatasa, Voges Proskawer, Optoquina, Fosfatasa, Bilis Esculina, Pirridolina, Arginina, Galactosidasa, Urea, Manitol, Lactosa, Trehalosa, Mannosa, Cloruro De Sodio, Sorbitol, Arabinosa, Ribosa, Inulina, Raffinosa, Bacitracina y Piruvato.

\section{Estudio de sensibilidad a los antibióti- cos y detección fenotípica de la produc- ción de $\beta$-lactamasas}

La evaluación de la sensibilidad antimicrobiana de las bacterias entéricas se realizó mediante el método de difusión del disco sobre placas con agar Müller Hinton (Oxoid Ltd., Hampshire, United Kingdom) utilizando un estándar de 0.5 Mcfarland. Los sensidiscos correspondieron a los siguientes antibióticos: cefotaxima (CTX $30 \mu \mathrm{g}$ ), ceftazidime (CAZ $30 \mu \mathrm{g}$ ), cefepime (FEP $30 \mu \mathrm{g}$ ), cefoxitina (FOX $75 \mu \mathrm{g}$ ), ceftriaxona $($ CRO $30 \mu \mathrm{g})$, piperacillina-tazobactam (TZP $10 \mu \mathrm{g} / 30 \mu \mathrm{g}$ ), aztreonam (ATM 30 $\mu \mathrm{g}$ ), imipenem (IMP $10 \mu \mathrm{g}$ ), meropenem (MEM $10 \mu \mathrm{g}$ ), gentamicina (GEN10 $\mu \mathrm{g}$ ), trimetoprim/sulfametoxazol (SXT $25 \mu \mathrm{g}$ ), levofloxacina (LEV $5 \mu \mathrm{g}$ ) (Oxoid Ltd., 
Hampshire, United Kingdom) de acuerdo con los lineamientos establecidos por el Clinical and Laboratory Standards Institute (CLSI) (17).

La susceptibilidad intermedia a cada antibiótico se consideró como resistencia. El control de calidad se realizó utilizando cepas estándar de Escherichia coli (ATCC 25922) y Pseudomonas aeruginosa (ATCC 27853).

Los aislamientos que fueron resistentes a aztreonam, cefotaxime, ceftazidime ó ceftriaxona fueron sometidos a la confirmación de presencia de $\beta$-lactamasa de espectro extendido (BLEE) mediante la prueba de sinergia de doble disco. En esta prueba, se colocó un disco de amoxicilina/ácido clavulánico $(20 \mu \mathrm{g} / 10 \mu \mathrm{g})$ en el centro de la placa y a $20 \mathrm{~mm}$ (centro a centro) lejos de un disco de aztreonam $(30 \mu \mathrm{g})$ y de un disco de ceftazidima $(30 \mu \mathrm{g})$. La placa se incubó a $35^{\circ} \mathrm{C}$ durante 24 horas. La prueba se consideró positiva cuando hubo un aumento o distorsión de la zona de inhibición entre cualquier marcador antimicrobiano y el disco de amoxicilina/ácido clavulánico.

Los aislamientos que fueron sensibles a la cefoxitina en el antibiograma se sometieron a la prueba confirmatoria de $\beta$-lactamasa cromosómica inducible, $\mathrm{AmpC}$, en el que se colocó un disco de cefoxitina $(30 \mu \mathrm{g})$ en el centro de la placa, a $20 \mathrm{~mm}$ (centro a centro) de un disco de ceftriaxona $(30 \mu \mathrm{g})$ y de un disco de ceftazidima $(30 \mu \mathrm{g})$. La placa se incubó a $35^{\circ} \mathrm{C}$ durante $18-24 \mathrm{~h}$. La cefoxitina funciona como un inductor de la enzima AmpC y la lectura se consideró positiva cuando se observó achatamiento del halo alrededor de la ceftriaxona y/o el disco de ceftazidima.

\section{Análisis fenotípico de la resistencia a los antibióticos de los EGV}

Para los EGV se evaluó la concentración inhibitoria mínima (CIM) de penicilina $(\mathrm{PEN})$, ceftriaxona (CRO), cefotaxime (CTX), cefepime (FEP), imipenem (IPM), tetraciclina (TET), levofloxacina (LEV), vancomicina (VAN), eritromicina (ERY), clindamicina (CLI), linezolid (LZD) y gentamicina (GEN) (Oxoid Ltd., Hampshire, United Kingdom) por dilución seriada en agar Mueller-Hinton suplementada con un $5 \%$ de sangre de carnero según los parámetros de CLSI (18).

El fenotipo de resistencia a macrólidos se determinó mediante discos comerciales de eritromicina $(15 \mu \mathrm{g})$ y clindamicina $(2 \mu \mathrm{g})$ (Oxoid Ltd., Hampshire, United Kingdom) colocados a una distancia de $12 \mathrm{~mm}$ sobre placas de agar Mueller-Hinton (Oxoid Ltd., Hampshire, United Kingdom) suplementada con un $5 \%$ de sangre de carnero e incubados en una atmósfera de $\mathrm{CO} 2$ a 37 oC durante 2 días.

La resistencia en ambos discos se consideró una resistencia constitutiva (cMLS $\beta$ ), mientras que el achatamiento de la zona de inhibición del disco de clindamicina en 
la zona próxima al disco de eritromicina indicó resistencia inducible (iMLS $\beta$ ). La sensibilidad detectada a clindamicina sin achatamiento en la zona de inhibición se consideró un fenotipo M. El fenotipo L se definió cuando se observó resistencia en el disco de clindamicina y sensibilidad a eritromicina.

Aislamiento de DNA de las cepas referencia y de los aislados bacterianos

El DNA de las bacterias entéricas y los EGV se obtuvo empleando el kit "Easy-DNATM" (Invitrogen, life technologies). La calidad y concentración del ADN se determinó por espectrofotometría usando el equipo nanodrop (Thermo $2000^{\circ}$ ).

Amplificación por PCR de los determinantes genéticos que confieren resistencia a los antibióticos

Para la identificación de genes bla se amplificó 5 a $10 \eta$ g de ADN genómico con 2,5 pmol de cada cebador para amplificar los genes blaIMP-1(18), blaVIM-2(19), blaTEM-1 (20), blaCTXM-9 (21), blaOXA-10 (22) y blaampC (23) y se mezcló con $0,2 \mathrm{mM}$ dNTPs (BioLine $\left.{ }^{\oplus}\right), 1,5 \mathrm{mM}$ de $\mathrm{MgCl} 2$ y 0,6 U de Taq polimerasa (Invitrogen $^{\circledast}$ ) en un volumen final de $25 \mu$ l. Las condiciones de amplificación fueron: denaturación a $94 \mathrm{oC}$ por 4 minutos, seguidos por 30 ciclos a $940 \mathrm{C}$ por 45 segundos, una temperatura de alineamiento a $67^{\circ} \mathrm{C}$ por 45 segundos, extensión a $720 \mathrm{C}$ por 1 minuto y una extensión final a $72^{\circ} \mathrm{C}$ por 10 minutos. La temperatura de alineamiento para los genes blaCTX-M-9 y blaOXA-10 fue de $60 \mathrm{oC}$.

La amplificación por PCR de los determinantes de resistencia a eritromicina se realizó empleando los conjuntos de cebadores ermA, ermB, mef y mphA de acuerdo con el protocolo establecido por Sutcliffe et al (14). La secuencia de los cebadores se encuentra en la Tabla 1. 
Tabla 1. Secuencia nucleotídicas de los cebadores empleados en este estudio

\begin{tabular}{|c|c|c|c|}
\hline Cebador & Secuencia del oligonicleótido $\left(5^{\prime} \rightarrow 3\right)$ & tamaño $(\mathrm{pb})$ & Referencia \\
\hline blaTEM-1 & $\begin{array}{l}\text { F-5'ATGAGTATTCAACAT TTC CG3' } \\
\text { R-5'CTG ACA GTT ACC AAT GCT TA3' }\end{array}$ & 956 & 21 \\
\hline bla VIM-2 & $\begin{array}{l}\text { F-5'AAAGTTATGCCGCACTCACC3' } \\
\text { R-5'TGCAACTTCATGTTATGCCG3' }\end{array}$ & 865 & 20 \\
\hline bla IMP-1 & $\begin{array}{l}\text { F- 5'ATGAGCAAGTTATCCTTATTC3' } \\
\text { R- 5'GCTGCAACGACTTGTTAG3' }\end{array}$ & 741 & 19 \\
\hline blaCTX-M-9 & $\begin{array}{l}\text { F -5'GTGACAAAGAGAGTGCAACGG3' } \\
\text { R-5'ATGATTCTCGCCGCTGAAGCC-3' }\end{array}$ & 856 & 22 \\
\hline blaOXA-10 & $\begin{array}{l}\text { F5'-TGAGCACCATAAGGCAACCA-3' } \\
\text { 'R5'-TTGGGCTAAATGGAAGCGTTT-3' }\end{array}$ & 311 & 23 \\
\hline BlaampC & $\begin{array}{l}\text { F- GGTATGGCTGTGGGTGTTA-3’ } \\
\text { R-5’ TCCGAAACGGTTAGTTGAG 3’' }\end{array}$ & 882 & 24 \\
\hline ermA & $\begin{array}{l}\text { F5' AACACCCTGAACCCAAGGGACG- 3' } \\
\text { R5' CTTCACATCCGGATTCGCTCGA 3' }\end{array}$ & 420 & 16 \\
\hline ermB & $\begin{array}{l}\text { F5' -AGAAATGGAGGTTCATACTTACCA-3' } \\
\text { R5' CATATAATCATCACCAATGGCA-3' }\end{array}$ & 546 & 16 \\
\hline$m p h A$ & $\begin{array}{l}\text { F5' -AACTGTACGCACTTGC--3' } \\
\text { R5' GGTACTCTTCGTTACC-3' }\end{array}$ & 837 & 16 \\
\hline mef & $\begin{array}{l}\text { mefA 5' - AGTATCATTAATCACTAGTGC--3' } \\
\text { mefE 5' TTCTTCTGGTACTAAAAGTGG-3' }\end{array}$ & 348 & 16 \\
\hline
\end{tabular}

Fuente: Autores.

Se realizó una electroforesis en geles de agarosa al 1,2\% para distinguir los productos de la PCR. El tamańo de cada producto de PCR se estimó utilizando marcadores de peso molecular estándar (escala de ADN de $1 \mathrm{~kb}$ de Gibco BRL, Gaithersburg, Md.) y se reveló empelando SYBR Green (Bio-Rad) bajo luz UV en el transiluminador.

\section{Análisis estadísticos de los resultados}

La frecuencia de aislamientos con resistencia a los antibióticos se calculó como la prevalencia de pacientes con aislamientos resistente a los antibióticos. La diferencia en la frecuencia de las variables y su asociación fueron determinadas por análisis de $\mathrm{X}$, un valor de $\mathrm{p} \leq 0,05$ se consideró estadísticamente significativo. Para realizar este análisis se empleó el paquete estadístico SPSS Vs.23 (Inc, Chicago, IL).

\section{Consideraciones éticas}

Este estudio fue respaldado por el Comité de la Clínica Odontológica (Ley Núm. 05-013). Todos los pacientes estaban suficientemente informados sobre los objetivos y procedimientos. Se obtuvieron muestras y datos después de firmar el consentimiento informado por escrito. El estudio cumplió con las disposiciones de la Declaración de 
Helsinki y las normas científicas técnicas y administrativas para la investigación en salud mencionadas en la "Resolución 8430 de 1993" del Ministerio de Salud de Colombia.

\section{Resultados}

Un total de 193 aislamientos fueron obtenidos, 59 aislamientos correspondieron a bacilos entéricos y 134 fueron EGV.

Entre los bacilos entéricos se encontró que el mayor número de aislamientos correspondió a E. coli con el 32,2\% (19/59), seguido por Klebsiella oxytoca con el $22 \%$ $(13 / 59)$ y $K$. pneumoniae con el $15,3 \%$ (9/59). También se aisló Enterobacter claocae, Citrobacter freundii, E. aerogenes y Proteus mirabilis en menor proporción (ver Tabla 2).

\section{Análisis de los perfiles de sensibilidad en los aislamientos de bacilos entéricos}

Más del 47\% de los aislamientos de los bacilos entéricos presentaron resistencia a los antibióticos evaluados. Cifras cercanas al 90\% o más altas se encontraron de aislamientos resistentes a cefalosporinas de tercera generación como cefoperazona $(98,3 \%)$, cefoxitin $(91,5 \%)$ y cefotaxima $(89,9 \%)$ y a aztreonam (88\%). Los aislamientos resistentes a carbapenemes y a cefalosporinas de cuarta generación estuvieron por encima del 45\% (ver Tabla 2). E. coli presentó el mayor número de aisalmientos con resisten- cia especialmente a cefepime $(46,4 \%)$, ceftriaxona $(38,8 \%)$ y carbapenemes $(37,1 \%)$. Los aislamientos de $K$. oxytoca y $K$. pneumoniae mostraron también resistencia a los antibióticos $\beta$-lactámicos, especialmente a las cefalosporina y carbapenemes como se observa en la Tabla 2. 
Tabla 2. Perfíl de sensibilidad a los antibióticos en los aislamientos de bacilos entéricos de cavidad oral. $\mathrm{n}=59$.

\begin{tabular}{|c|c|c|c|c|c|c|c|c|c|c|c|c|}
\hline \multirow{2}{*}{ Aislamientos } & \multicolumn{12}{|c|}{ Antibiograma } \\
\hline & $\begin{array}{c}\text { TZP } \\
\text { n (\%) }\end{array}$ & $\begin{array}{r}\text { CAZ } \\
\text { n (\%) }\end{array}$ & $\begin{array}{l}\text { CTX } \\
\text { n (\%) }\end{array}$ & $\begin{array}{l}\text { FOX } \\
\text { n (\%) }\end{array}$ & $\begin{array}{l}\text { CRO } \\
\text { n (\%) }\end{array}$ & $\begin{array}{l}\text { FEP } \\
\text { n (\%) }\end{array}$ & $\begin{array}{l}\text { ATM } \\
\text { n (\%) }\end{array}$ & $\begin{array}{l}\text { MEM } \\
\text { n (\%) }\end{array}$ & $\begin{array}{l}\text { IMP } \\
\text { n (\%) }\end{array}$ & $\begin{array}{l}\text { LEV } \\
\text { n (\%) }\end{array}$ & $\begin{array}{l}\text { SXT } \\
\text { n (\%) }\end{array}$ & $\begin{array}{l}\text { GEN } \\
\text { n (\%) }\end{array}$ \\
\hline $\begin{array}{c}\text { Escherichia coli } \\
n=19\end{array}$ & $\begin{array}{c}17 \\
(34)\end{array}$ & $\begin{array}{c}19 \\
(35,2)\end{array}$ & $\begin{array}{c}19 \\
(35,8)\end{array}$ & $\begin{array}{c}19 \\
(32,8)\end{array}$ & $\begin{array}{c}19 \\
(38,8)\end{array}$ & $\begin{array}{c}13 \\
(46,4)\end{array}$ & $\begin{array}{c}19 \\
(36,5)\end{array}$ & $\begin{array}{c}10 \\
(37)\end{array}$ & $\begin{array}{c}13 \\
(37,1)\end{array}$ & $\begin{array}{c}10 \\
(26,3)\end{array}$ & $\begin{array}{c}15 \\
(39,5)\end{array}$ & $\begin{array}{c}9 \\
(31)\end{array}$ \\
\hline $\begin{array}{c}\text { Klebsiella oxytica } \\
\quad n=13\end{array}$ & $\begin{array}{l}13 \\
(26)\end{array}$ & $\begin{array}{c}13 \\
(24,1)\end{array}$ & $\begin{array}{c}12 \\
(22,6)\end{array}$ & $\begin{array}{c}13 \\
(22,4)\end{array}$ & $\begin{array}{c}12 \\
(24,5)\end{array}$ & $\begin{array}{c}6 \\
(21,4)\end{array}$ & $\begin{array}{c}10 \\
(19,2)\end{array}$ & $\begin{array}{c}10 \\
(37)\end{array}$ & $\begin{array}{c}5 \\
(14,3)\end{array}$ & $\begin{array}{c}6 \\
(15,8)\end{array}$ & $\begin{array}{c}6 \\
(15,8)\end{array}$ & $\begin{array}{c}7 \\
(24,1)\end{array}$ \\
\hline $\begin{array}{c}\text { Klebsiella } \\
\text { pneumoniae } n=9\end{array}$ & $\begin{array}{c}7 \\
(14)\end{array}$ & $\begin{array}{c}7 \\
(13)\end{array}$ & $\begin{array}{c}9 \\
(17)\end{array}$ & $\begin{array}{c}9 \\
(15,5)\end{array}$ & $\begin{array}{c}8 \\
(16,3)\end{array}$ & $\begin{array}{c}8 \\
(28,6)\end{array}$ & $\begin{array}{c}8 \\
(15,4)\end{array}$ & $\begin{array}{c}5 \\
(18,5)\end{array}$ & $\begin{array}{c}8 \\
(22,9)\end{array}$ & $\begin{array}{c}9 \\
(23,7)\end{array}$ & $\begin{array}{c}8 \\
(21,1)\end{array}$ & $\begin{array}{c}8 \\
(27,6)\end{array}$ \\
\hline $\begin{array}{l}\text { Enterobacter claocae } \\
\qquad n=5\end{array}$ & $\begin{array}{c}4 \\
(8)\end{array}$ & $\begin{array}{c}4 \\
(7,4)\end{array}$ & $\begin{array}{c}3 \\
(5,7)\end{array}$ & $\begin{array}{c}5 \\
(8,6)\end{array}$ & $\begin{array}{c}3 \\
(6,1)\end{array}$ & - & $\begin{array}{c}5 \\
(9,6)\end{array}$ & - & $\begin{array}{c}4 \\
(11,4)\end{array}$ & $\begin{array}{c}5 \\
(13,2)\end{array}$ & $\begin{array}{c}3 \\
(7,9)\end{array}$ & $\begin{array}{c}1 \\
(3,4)\end{array}$ \\
\hline $\begin{array}{l}\text { Citrobacter } \\
\text { freundii } n=3\end{array}$ & 3 & $\begin{array}{c}3 \\
(5,6)\end{array}$ & $\begin{array}{c}1 \\
(1,9)\end{array}$ & $\begin{array}{c}3 \\
(5,2)\end{array}$ & $\begin{array}{c}1 \\
(2)\end{array}$ & - & $\begin{array}{c}2 \\
(3,8)\end{array}$ & $\begin{array}{c}2 \\
(7,4)\end{array}$ & - & $\begin{array}{c}3 \\
(7,9)\end{array}$ & $\begin{array}{c}2 \\
(5,3)\end{array}$ & - \\
\hline $\begin{array}{l}\text { Enterobacter } \\
\text { aerogenes } n=2\end{array}$ & $\begin{array}{c}1 \\
(0,2)\end{array}$ & $\begin{array}{c}2 \\
(3,7)\end{array}$ & $\begin{array}{c}2 \\
(3,8)\end{array}$ & $\begin{array}{c}2 \\
(3,4)\end{array}$ & $\begin{array}{c}2 \\
(4,1)\end{array}$ & $\begin{array}{c}1 \\
(3,6)\end{array}$ & $\begin{array}{c}2 \\
(3,8)\end{array}$ & - & - & $\begin{array}{c}2 \\
(5,3)\end{array}$ & $\begin{array}{c}1 \\
(2,6)\end{array}$ & - \\
\hline $\begin{array}{c}\text { Proteus mirabilis } \\
n=2\end{array}$ & $\begin{array}{c}1 \\
(0,2)\end{array}$ & $\begin{array}{c}1 \\
(1,9)\end{array}$ & $\begin{array}{c}2 \\
(3,8)\end{array}$ & $\begin{array}{c}2 \\
(3,4)\end{array}$ & $\begin{array}{c}2 \\
(4,1)\end{array}$ & - & $\begin{array}{c}2 \\
(3,8)\end{array}$ & - & - & - & $\begin{array}{c}1 \\
(2,6)\end{array}$ & $\begin{array}{c}1 \\
(3,4)\end{array}$ \\
\hline $\begin{array}{c}\text { Otras entreobacterias } \\
n=6\end{array}$ & $\begin{array}{c}4 \\
(8)\end{array}$ & $\begin{array}{c}5 \\
(9,3)\end{array}$ & $\begin{array}{c}5 \\
(9,4)\end{array}$ & $\begin{array}{c}4 \\
(6,7)\end{array}$ & $\begin{array}{c}2 \\
(4,1)\end{array}$ & - & $\begin{array}{c}4 \\
(7,7)\end{array}$ & - & $\begin{array}{c}5 \\
(14,3)\end{array}$ & $\begin{array}{c}3 \\
(7,9)\end{array}$ & $\begin{array}{c}2 \\
(5,3)\end{array}$ & $\begin{array}{c}3 \\
(10,3)\end{array}$ \\
\hline TOTAL & $\begin{array}{c}50 \\
(84,7)\end{array}$ & $\begin{array}{c}54 \\
(91,5)\end{array}$ & $\begin{array}{c}53 \\
(89,8)\end{array}$ & $\begin{array}{c}58 \\
(98,3)\end{array}$ & $\begin{array}{c}49 \\
(83,1)\end{array}$ & $\begin{array}{c}28 \\
(47,5)\end{array}$ & $\begin{array}{c}52 \\
(88,1)\end{array}$ & $\begin{array}{c}27 \\
(45,8)\end{array}$ & $\begin{array}{c}35 \\
(59,3)\end{array}$ & $\begin{array}{c}38 \\
(64,4)\end{array}$ & $\begin{array}{c}38 \\
(64,4)\end{array}$ & $\begin{array}{c}29 \\
(49,2)\end{array}$ \\
\hline
\end{tabular}

Fuente: Autores.

La Tabla 3 muestra los resultados del análisis de la prueba de sensibilidad a los antibióticos. Se determinaron 6 perfiles de sensibilidad (antibiotipos) entre los bacilos entéricos.

De acuerdo con la definición propuesta por Magiorakos et al (24), la resistencia a tres o más clases de antibióticos se definió como multirresistente (MDR). La mayoría de los bacilos entéricos presentan perfiles de MDR a los antibióticos; así, el 64,4\% de los aislamientos se ubicaron en el antibiotipo 5, con aislamientos MDR resistentes a 6 o 7 antibióticos. En este grupo se identificaron 15 aislamientos de E. coli y 9 aislamientos de $K$. oxytoca. Sin embargo, el antibiotipo 6 agrupó los bacilos con resistencia a todos los antibióticos evaluados (20,3\%). En este caso, seis aislamientos correspondieron a $E$. coli, cuatro a $K$. oxytoca y dos a $K$. pneumaniae.

Los antibiotipos 3 y 4 con perfiles de resistencia a 4 y 5 antibióticos, respectivamente se determinaron en el $5,1 \%$ de los aislamientos. Los aislamientos con los antibiotipos 1 y 2 fueron lo menos prevalentes.

En este estudio se detectaron fenotípicamente 39 aislamientos portadores de $\beta$-lactamasas. Esta detección tuvo en cuenta tres 
tipos de estas enzimas: $\beta$-lactamasas de espectro extendido (BLEE), $\beta$-lactamasas del tipo AmpC y carbapenemasas.

Los aislamientos que presentaron el fenotipo compatible con BLEE correspondieron al 20,5\% (8/39) y el fenotipo específico BLEE tipo CTX (detectado en seis aislamientos con resistencia a cefotaxima) correspondió al 15,4\%.
La $\beta$-lactamasa tipo AmpC se encontró en el 12,8\% (5/39) de los aislamientos, dos de ellos en $K$. Oxytoca, otros dos en $K$. pneumoniae y uno de $E$. aerogenes. Sin embargo, el $66,7 \%$, de los aislamientos presentaron el fenotipo carbapenemasas, representados principalmente por E. coli (17/39) y $K$. pneumoniae (7/39) (ver Tabla 4).

Tabla 3. Perfíl de sensibilidad a los antibióticos en los aislamientos de bacilos entéricos de cavidad oral. $\mathrm{n}=59$.

\begin{tabular}{|c|c|c|c|c|c|c|c|c|c|}
\hline \multirow{2}{*}{ Ant } & \multirow{2}{*}{$\begin{array}{c}\text { Aislamientos } \\
n \\
(\%)\end{array}$} & \multicolumn{6}{|c|}{ Tipo de gen bla } & \multirow{2}{*}{ Perfil de resistencia } & \multirow{2}{*}{ Perfil de sensibilidad } \\
\hline & & oxa-10 & tem-1 & imp-1 & vim-2 & ctx-9 & ampC & & \\
\hline 1 & $\begin{array}{c}2 \\
(3,4)\end{array}$ & - & - & - & - & - & - & CAZ, FOX & $\begin{array}{l}\text { TZP, CTX, CRO, } \\
\text { FEP, ATM, MEM, } \\
\text { IMP, LVX, SXT, } \\
\text { GEN }\end{array}$ \\
\hline 2 & $\begin{array}{c}1 \\
(1,7)\end{array}$ & - & - & - & - & - & - & CAZ, FOX, CRO & $\begin{array}{l}\text { TZP, CTX, FEP, } \\
\text { ATM, MEM, IMP, } \\
\text { LVX, SXT, GEN }\end{array}$ \\
\hline 3 & $\begin{array}{c}3 \\
(5,1)\end{array}$ & - & - & - & - & - & $\begin{array}{c}3 \\
(5,1)\end{array}$ & $\begin{array}{l}\text { TZP, ATM, FOX, } \\
\text { CRO, }\end{array}$ & $\begin{array}{l}\text { CTX, FEP, ATM, } \\
\text { MEM, IMP, LVX, } \\
\text { SXT, GEN }\end{array}$ \\
\hline 4 & $\begin{array}{c}3 \\
(5,1)\end{array}$ & - & $\begin{array}{c}1 \\
(1,7)\end{array}$ & - & - & $\begin{array}{c}1 \\
(1,7)\end{array}$ & $\begin{array}{c}2 \\
(3,4)\end{array}$ & $\begin{array}{l}\text { TZP, CAZ, CTX, } \\
\text { FOX, SXT/ ATM }\end{array}$ & $\begin{array}{l}\text { CRO, FEP, MEM, } \\
\text { IMP, LVX, GEN - }\end{array}$ \\
\hline 5 & $\begin{array}{c}38 \\
(64,4)\end{array}$ & $\begin{array}{c}4 \\
(6,8)\end{array}$ & $\begin{array}{c}19 \\
(32,2)^{*}\end{array}$ & $\begin{array}{c}1 \\
(1,7)\end{array}$ & $\begin{array}{c}11 \\
(18,6)^{*}\end{array}$ & $\begin{array}{c}5 \\
(8,5)\end{array}$ & & $\begin{array}{l}\text { TZP, CAZ, CTX, } \\
\text { FOX, ATM,CRO/ } \\
\text { FEP, MEM/ IMP, } \\
\text { LVX/ SXT }\end{array}$ & $\begin{array}{c}\text { GEN, CRO/FEP, } \\
\text { MEM/ IMP, LVX/ } \\
\text { SXT }\end{array}$ \\
\hline 6 & $\begin{array}{c}12 \\
(20,3)\end{array}$ & - & $\begin{array}{c}9 \\
(15,3)^{*}\end{array}$ & $\begin{array}{c}3 \\
(5,1)\end{array}$ & $\begin{array}{c}7 \\
(11,9)^{*}\end{array}$ & - & & $\begin{array}{l}\text { TZP, CAZ, CTX, } \\
\text { FOX, CRO, FEP, } \\
\text { ATM: MEM, IMP, } \\
\text { LVX, SXT, GEN }\end{array}$ & - \\
\hline
\end{tabular}

Fuente: Autores.

\section{Detección de los genes bla}

La distribución de los genes bla entre los antibiotipos y los fenotipos detectados en los aislamientos de los bacilos entéricos se encuentra en la Tabla 3 y 4 , respectivamente.
El mayor número de aislamientos presentaron el blaTEM-1 (49,2\%). Este gen codifica para una $\beta$-lactamasa de amplio expectro (BLEA).

Es importante destacar que en el $30,5 \%$ de los aislamientos se detectó el gen bla- 
VIM-2, el cual codifica por una Metalo- $\beta$-lactamasa y confiere resistencia a carbapenemes. En concordancia con este resultado, se observó que la presencia de este gen fue significativa entre aquellos aislamientos que presentaron el fenotipo carbapenemasa $(\mathrm{p}<0,05)$.

En este estudio también fue significativa la presencia de blaCTX-M-9 en los aislamientos con el fenotipo BLEE (ver Tabla 4).

Los genes bla se detectaron sólo en aquellos aislamientos con un fenotipo MDR. Los bacilos entéricos con el antibiotipo 5 presentaron todos los genes bla, excepto el blampC, siendo el gen blaTEM-1 con el $32,2 \%$, el más abundante, seguido del gen blaVIM-2 (18,6\%). Los bacilos entéricos con el antibiotipo 6 presentaron también abundancia de genes bla. El gen blaampC fue determinado sólo en los bacilos con los antibiotipo 3 y 4 (ver Tabla 3).
Al establecer la concordancia de los genes bla con el fenotipo determinado en la Tabla 4, encontramos que el 10,2\% de los bacilos entéricos con el fenotipo BLEE presentaron el gen blaCTX-M-9 y el 6,8\% blaTEM-1.

Todas las cepas con el fenotipo AmpC presentaron los genes blaTEM-1 y blampC. En el caso de los bacilos entéricos con carbapenemasas, se encontró que la resistencia a estos antibióticos se debió principalmente por la presencia de genes que codifican para las metalo enzimas blaVIM-2 $(30,5 \%)$ y blaIMP-1 (6,8\%), siendo significativa la presencia de cepas con el gen blaVIM-2 ( $\mathrm{p}<0,05)$. En el 6,8\% de estas cepas se determinó que la resistencia a los carbapenemes estaría mediada por el gen blaOXA-10. Algunas cepas presento más de un gen bla.

Tabla 4. Distribución de los aislamientos de bacilos entéricos Gram negativos con el fenotipo $\beta$-lactamasas $(n=39)$ y de los genes bla.

\begin{tabular}{|c|c|c|c|c|c|c|}
\hline Fenotipo & \multicolumn{6}{|c|}{ Tipo de gen bla } \\
\hline $\begin{array}{c}\text { Tipo de } \\
\beta \text {-lactamasas }\end{array}$ & $\begin{array}{c}\text { blaTEM-1 } \\
n \\
(\%)\end{array}$ & $\begin{array}{c}\text { blaCTX-M-9 } \\
n \\
(\%)\end{array}$ & $\begin{array}{c}\text { blaOXA-10 } \\
n \\
(\%)\end{array}$ & $\begin{array}{c}\text { blaVIM-2 } \\
n \\
(\%)\end{array}$ & $\begin{array}{c}\text { bla IMP-1 } \\
n \\
(\%)\end{array}$ & $\begin{array}{c}\text { blaampC } \\
n \\
(\%)\end{array}$ \\
\hline BLEE $n=8$ & $\begin{array}{c}4 \\
(6,8)\end{array}$ & $\begin{array}{c}6 \\
(10,2)^{*}\end{array}$ & - & - & - & - \\
\hline $\begin{array}{c}\text { Carbapenemasas } \\
n=26\end{array}$ & $\begin{array}{c}20 \\
(33,9)^{*}\end{array}$ & - & $\begin{array}{c}4 \\
(6,8)\end{array}$ & $\begin{array}{c}18 \\
(30,5)^{*}\end{array}$ & $\begin{array}{c}4 \\
(6,8)\end{array}$ & - \\
\hline $\operatorname{ampC} n=5$ & $\begin{array}{c}5 \\
(8,5)\end{array}$ & - & - & - & - & $\begin{array}{c}5 \\
(8,5)\end{array}$ \\
\hline Total & $\begin{array}{c}29 \\
(49,2)\end{array}$ & $\begin{array}{c}6 \\
(10,2)\end{array}$ & - & $\begin{array}{c}18 \\
(30,5)\end{array}$ & $\begin{array}{c}4 \\
(6,8)\end{array}$ & $\begin{array}{c}5 \\
(8,5)\end{array}$ \\
\hline
\end{tabular}

Fuente: Autores. 
Análisis fenotípico y molecular de los aislamientos de los EGV

Entre los aislamientos de los EGV, el $S$. $s a$ livarius fue el más común, seguido de $S$.anginosus, S. mitis, S. sanguinis, S. bovis, y $S$. mutans.

El 74,6\% (100/134) fueron resistentes a penicilina, $14,2 \%(19 / 134)$ a ceftriaxona, $4,5 \%(6 / 134)$ a gentamicina, $11,2 \%$
$(15 / 134)$ a tetraciclina, $38,8 \%(52 / 134)$ a eritromicina y el $28,4 \%(38 / 134)$ a clindamicina. El resto de los antibióticos evaluados presentaron $100 \%$ de actividad contra los aislamientos de EGV (ver Tabla 5).

S. salivarius presentó el mayor número de aislamientos con resistencia a penicilina (53\%) y S. anginosus a eritromicina y clindamicina $(53,8 \%$ y $52,6 \%$, respectivamente).

Tabla 5. Perfil de sensibilidad a los antibióticos en los aislamientos de los Streptococcus del grupo viridans. $\mathrm{n}=134$.

\begin{tabular}{|c|c|c|c|c|c|c|}
\hline \multirow{2}{*}{ Aislamientos } & \multicolumn{6}{|c|}{ Antibiograma } \\
\hline & $\begin{array}{l}\text { PEN } \\
\text { n (\%) }\end{array}$ & $\begin{array}{r}\text { CTX } \\
\text { n (\%) }\end{array}$ & $\begin{array}{r}\text { TET } \\
\text { n (\%) }\end{array}$ & $\begin{array}{c}\text { ERY } \\
\text { n (\%) }\end{array}$ & $\begin{array}{c}\text { CLI } \\
\text { n (\%) }\end{array}$ & $\begin{array}{l}\text { GEN } \\
\text { n(\%) }\end{array}$ \\
\hline $\begin{array}{c}\text { S. salivarius } \\
n=62\end{array}$ & $\begin{array}{c}53 \\
(53)\end{array}$ & $\begin{array}{c}6 \\
(31,6)\end{array}$ & $\begin{array}{c}5 \\
(33,3)\end{array}$ & $\begin{array}{c}12 \\
(23,1)\end{array}$ & $\begin{array}{c}11 \\
(28,9)\end{array}$ & $\begin{array}{c}1 \\
(16,7)\end{array}$ \\
\hline $\begin{array}{c}\text { S. anginosus } \\
n=34\end{array}$ & $\begin{array}{c}22 \\
(22)\end{array}$ & $\begin{array}{c}3 \\
(15,8)\end{array}$ & $\begin{array}{c}2 \\
(13,3)\end{array}$ & $\begin{array}{c}28 \\
(53,8)\end{array}$ & $\begin{array}{c}20 \\
(52,6)\end{array}$ & $\begin{array}{c}3 \\
(50)\end{array}$ \\
\hline $\begin{array}{c}\text { S. mitis } \\
n=20\end{array}$ & $\begin{array}{c}14 \\
(14) \\
\end{array}$ & $\begin{array}{c}7 \\
(36,8) \\
\end{array}$ & $\begin{array}{c}5 \\
(33,3) \\
\end{array}$ & $\begin{array}{c}6 \\
(11,5) \\
\end{array}$ & $\begin{array}{c}6 \\
(15,8) \\
\end{array}$ & $\begin{array}{c}2 \\
(33,3) \\
\end{array}$ \\
\hline $\begin{array}{c}\text { S. sanguinis } \\
n=12\end{array}$ & $\begin{array}{c}7 \\
(7) \\
\end{array}$ & $\begin{array}{c}2 \\
(10,5) \\
\end{array}$ & $\begin{array}{c}2 \\
(13,3)\end{array}$ & $\begin{array}{c}5 \\
(9,6)\end{array}$ & - & - \\
\hline $\begin{array}{c}\text { S. bovis } \\
n=5\end{array}$ & $\begin{array}{c}3 \\
(3)\end{array}$ & $\begin{array}{c}1 \\
(5,3)\end{array}$ & - & $\begin{array}{c}1 \\
(1,9)\end{array}$ & - & - \\
\hline $\begin{array}{c}\text { S. mutans } \\
n=1\end{array}$ & $\begin{array}{c}1 \\
(1) \\
\end{array}$ & - & $\begin{array}{c}1 \\
(6,7) \\
\end{array}$ & - & - & - \\
\hline TOTAL & $\begin{array}{c}100 \\
(74,6)\end{array}$ & $\begin{array}{c}19 \\
(14,2)\end{array}$ & $\begin{array}{c}15 \\
(11,2)\end{array}$ & $\begin{array}{c}52 \\
(38,8)\end{array}$ & $\begin{array}{c}38 \\
(28,4)\end{array}$ & $\begin{array}{c}6 \\
(4,5)\end{array}$ \\
\hline
\end{tabular}

Fuente: Autores.

Los resultados de la evaluación fenotípica de la resistencia a eritromicina y clindamicina fueron los siguientes: $18,7 \%$ de los aislamientos (25/134) presentaron un fenotipo cMLS $\beta$ de resistencia constitutivo, el $4.5 \%(6 / 134)$ un fenotipo iMLS $\beta$ inducible, el 14,9\% (20/134) un fenotipo M.
Aislamientos de EGV con el fenotipo L no fueron detectados.

En los aislamientos cMLS $\beta$, el gen ermB se determinó con mayor frecuencia (18/25), tres de estos aislamientos presentaron también el gen ermA y otros tres aislamientos 
el genes mef. Sin embargo, seis aislamientos cMLS $\beta$ presentaron únicamente el gen $m p h$ y un aislamiento el gen mef. con el fenotipo $\mathrm{M}$, sólo se logró determinar el gen mef en el 9,7\% (13/134) de ellos como se muestra en la tabla 6 .

Entre los aislamientos iMLS $\beta$ se determinó los genes mph y mef. Entre los aislamientos

Tabla 6. Distribución de los genes de resistencia a eritromicina entre los diferentes fenotipos de EGV.

\begin{tabular}{|c|c|c|c|c|c|}
\hline \multirow{2}{*}{\multicolumn{2}{|c|}{ Fenotipo }} & \multicolumn{4}{|c|}{ Genotipo } \\
\hline & & \multirow{2}{*}{$\begin{array}{l}\text { ermA } \\
\boldsymbol{n}(\%)\end{array}$} & \multirow{2}{*}{$\begin{array}{c}\text { ermB } \\
\boldsymbol{n}(\%)\end{array}$} & \multirow{2}{*}{$\begin{array}{c}m p h \\
\boldsymbol{n}(\%)\end{array}$} & \multirow{2}{*}{$\begin{array}{c}\begin{array}{c}m e f \\
n(\%)\end{array} \\
4(3.0)\end{array}$} \\
\hline cMLS $\beta$ & $\mathrm{n}=25$ & & & & \\
\hline iMLS $\beta$ & $n=6$ & - & - & $4(7,5)$ & $2(4.5)$ \\
\hline M & $\mathrm{n}=20$ & - & - & - & $13(9,7)$ \\
\hline
\end{tabular}

Fuente: Autores.

\section{Discusión}

Los bacilos entéricos Gram negativos pueden habitar la cavidad oral bajo condiciones fisiológicas especiales. En este estudio, los aislamientos entéricos obtenidos de la saliva y placa dental de sujetos sanos fueron del $33,1 \%$. Este valor fue superior al 23,3\% y $18,7 \%$ encontrado en un estudio similar realizado con estudiantes (8) y trabajadores de un hospital en Sao Paulo-Brasil (9), respectivamente.

Bryskier et al, sugieren que la prevalencia de los bacilos entéricos en la boca variaba de acuerdo a las características de los individuos: en pacientes con dentadura postiza tienen una prevalencia hasta del $48 \%$, en pacientes con hiatosis del $27,1 \%$, en los pacientes de ortodoncia del $13 \%$ y en pacientes con una cavidad oral sana del $16,4 \%$ (10). Según este resultado, nuestros hallazgos sólo fueron menores que en el caso de los sujetos con dentadura postiza.

Ferreira et al, establecen que la colonización de este nicho por los bacilos entéricos comienza tempranamente. Los recién nacidos presentan un $8 \%$ de colonización por estas bacterias, siendo Enterobacter spp. la más prevalente y en algunas cepas ya se detecta resistencia, especialmente a ampicilina, gentamicina y cefalotina (25).

En este estudio se evidenció diversidad de especies de Enterobacteriaceae en la cavidad oral de personas sin enfermedad dental 
con resistencia a los antibióticos $\beta$-lactámicos por encima del $80 \%$ de prevalencia, especialmente a las cefalosporinas de tercera generación, aztreonam y piperacilina/ tazobactam, siendo potenciales fuentes de infecciones oportunistas como lo han señalado algunos investigadores $(7,26,27)$.

El estudio desarrollado en jóvenes estudiantes en Brasil encontró que el 57,8\% y $45,3 \%$ de los bacilos entéricos mostraron resistencia a la amoxicilina/ácido clavulánico y cefoxitina, respectivamente; y fueron $100 \%$ sensibles a cefepime, ciprofloxacina, gentamicina, imipenem, meropenem y levofloxacina (8). En forma similar, Gaetti-Jardim et al reportan niveles significativos de resistencia para todos los $\beta$-lactámicos, excepto para imepenem y meropenem, que respectivamente presentaron $2,3 \%$ y $1,6 \%$ de resistencia (28).

Los resultados presentados en la Tabla 2 muestran que los carbapenemes y cefepime fueron los únicos $\beta$-lactámicos que tuvieron una actividad antimicrobiana en menos del 55\% de los aislamientos. La resistencia al imepenem fue levemente más alta a la observada al meropenem.

Los antibióticos $\beta$-lactámicos constituyen los agentes antimicrobianos más tradicionales empleados en el tratamiento de infecciones de todo tipo en Colombia, esto explicaría la alta frecuencia de enterobacterias resistentes halladas en este estudio. El aumento de la resistencia a estos antibióticos se debe a la producción de enzi- mas $\beta$-lactamasas principalmente. En este estudio encontramos que el $66,1 \%$ de los bacilos entéricos presentaron el fenotipo $\beta$-lactamasas, detectándose, tres tipos de ellas: BLEE, AmpC y carbapenemasas.

Los aislamientos productores de BLEEs detectados en la cavidad oral sana representaron el 13,6\%. La presencia de estas cepas es un problema importante en infecciones adquiridas en pacientes hospitalizados y en la comunidad porque producen desde una infección urinaria no complicada adquiri$\mathrm{da}$ en la comunidad hasta sepsis nosocomial potencialmente mortal.

Los aislamientos con el fenotipo AmpC representaron el $8,5 \%$ y fueron resistentes a la cefoxitina, lo que indica que son organismos mutantes y producen constantemente esta enzima independientemente de la exposición a un antibiotico inductor. Las infecciones debidas a este tipo de cepas, son difíciles de tratar debido al perfil MDR que presentan frente a los antibióticos $\beta$-lactámicos comúnmente empleados (29).

Es importante destacar que el más alto número de aislados fueron resistentes a los carbapenemes $(44,1 \%)$. Este tipo de antibiótico se emplea como última elección para el tratamiento de infecciones complicadas en las cuales los otros antibióticos $\beta$-lactámicos no han sido efectivos. Los genes encontrados entre los aislados resistentes a los carbapenemes fueron aquellos que codifican para $\beta$-lactamasas de la clase $B$, tipo IMP y VIM. 
Otro hallazgo importante fue la alta prevalencia del fenotipo MDR (84,7\%) entre los aislamientos agrupados con los antibiotipos 5 y 6. Además, estos aislamientos fueron portadores de genes bla, con predominio significativo de los genes blaTEM-1, y blaVIM-2.

Los reportes señalan que en la cavidad oral $y$ en el intestino del humano predominan principalmente $\beta$-lactamasas de la clase $A$, clase $\mathrm{C}$ y clase $\mathrm{D}$; mientras que las $\beta$-lactamasas de la clase $\mathrm{B}$ son más representativas de la microbiota del suelo $(1,3)$. Sin embargo, en este estudio encontramos un alto predominio de genes bla que codifica para $\beta$-lactamasas clase B.

Estos resultados evidencian que la cavidad oral sana es un reservorio de cepas bacterianas capaces de producir diferentes tipos de $\beta$-lactamasas y potenciales fuentes de genes bla que pueden propagarse a otras poblaciones como lo han establecido ya otros autores $(11,12,14,15)$.

En el caso de los aislamientos de EGV recuperados en el período de estudio, estos presentaron resistencia a algunos antibióticos $\beta$-lactámicos como la penicilina y ceftriaxona. También fue importante el número de aislamientos con resistencia a la eritromicina $(38,8 \%)$ y clindamicina $(28,4 \%)$. Estos resultados son concordantes con los reportados por otros estudios, con resistencias que van desde un 35\%-70\% para macrólidos y del 10-15\% para penicilinas (naturales o sintéticas) y clindamicina (4-6).
La alta resistencia a los macrólidos entre los aislamientos de EGV se asocia frecuentemente a una alta resistencia a las tetraciclinas, la clindamicina y los azálidos $(5,6)$.

En este estudio encontramos una correlación entre el fenotipo de resistencia a la eritromicina y el genotipo. Todos los aislamientos con el fenotipo cMLS $\beta$ presentaron los genes erm, principalmente el gen erm B lo que evidencia que la resistencia a eritromicina es mediada principalmente por metilación. En los Streptococcus spp. diversos investigadores han encontrado una relación importante entre la presencia del gen ermB y la expresión del fenotipo MLSB constitutivo $(16,30,31)$.

En relación al gen ermA es frecuente entre los aislamientos de Staphylococcus aureus resistente a eritromicina, con prevalencia mayor al 80\% (32). esto indicaría, quizás, que el gen ermA detectado en algunos aislamientos de EGV podrían ser fuente de $S$. aureus presentes en la cavidad oral de estas personas.

Es de destacar que entre los aislamientos iMLS $\beta$, cMLS $\beta$ y principalmente en los M, la resistencia a eritromicina se debió también a bombas de eflujo codificadas por el gen mef.

Los reportes indican el aumento en la expresión de la bomba de flujo codificada por este gen confiriendo resistencia a la acriflavina, aminoglucósidos, glicilciclina, macrólidos y $\beta$-lactámicos $(33,34)$. 
El tratamiento de la infección odontogénica genera una alta prescripción de antibióticos en la comunidad, aumentando la posibilidad de tener microrganismos resistentes. Sin embargo, algunos investigadores sugieren que la alta diversidad de organismos resistentes y la abundancia de genes de resistencia en la cavidad oral estaría siendo influenciada principalmente por factores ambientales generados durante los cambios estacionales y el tipo de alimentación como se ha demostrado en individuos japoneses (35), de países del suroeste de Europa como España, Francia e Italia (36) y Latinoamérica (37-38).

\section{Conclusión}

En este estudio se demostró la presencia de EVG y bacilos entéricos Gram negativos resistentes a los antibióticos y portadores de genes resistentes a eritromicina y genes bla, respectivamente. La presencia de estas bacterias en la cavidad oral sana representa un riesgo no solo para la salud de los individuos portadores, sino que contribuye a la creciente epidemia de resistencia bacteriana por varias implicaciones: 1) pueden considerarse como reserva genética a partir de la cual se puede transferir factores de resistencia a otras bacterias más patógenas; 2) tienen la potencialidad de producir enfermedades sistémicas difícil de tratar por el carácter de multirresistencia que presentan; y 3) tienen la potencialidad de desarrollar infecciones nosocomiales, si estos pacientes requieren en algún momento ser hospitalizados.

\section{Agradecimientos}

Los autores de este estudio agradecen a la Dirección de Investigaciones Facultad de Ciencias de la Salud de la Universidad Libre, Seccional Cali por el apoyo económico.

\section{Referencias}

1. Nesme J, Cecillon S, Delmont TO, Monier JM, Vogel TM, Simonet P. Large-scale metagenomic-based study of antibiotic resistance in the environment. Curr Biol. 2014;24:1096-1100. http://dx.doi.org/10.1016/j.cub.2014.03.036

2. Sommer MOA, Church GM, Dantas G. The human microbiome harbors a diverse reservoir of antibiotic resistance genes. Virulence. 2010; 1(4): 299-303. https://www.researchgate.net/publication/49702544

3. Penders J, Stobberingh EE, Savelkoul PH, Wolffs PF. The human microbiome as a reservoir of antimicrobial resistance. Front Microbiol. 2013;4:87. Published 2013 Apr 17. doi:10.3389/fmicb.2013.00087

4. Khalil D, Hultin M, Rashid MU, Lund B. Oral microflora and selection of resistance after a single dose of amoxicillin. Clin. Microbiol. Infect. 2016;22:949. e1-949.e4. doi:10.1016/j.cmi.2016.08.008

5. Chaffanel F, Charron-Bourgoin F, Libante V, Leblond-Bourget N, Payot S. Resistance genes and genetic elements associated with antibiotic resistance in clinical and commensal isolates of Streptococcus salivarius. Appl. Environ. Microbiol. 2015;81:41554163. doi: 10.1128/AEM.00415-15. Epub 2015 Apr 10

6. Pasquantonio G, Condo S, Cerroni L, Bikiqu L, Nicoletti M, Prenna M, Ripa S. Antibacterial activity of various antibiotics against oral streptococci isolated oral cavity Int J Immunopathol Pharmacol. 2012;25:805-809. doi: $10.1177 / 039463201202500331$ 
7. Dupin C, Tamanai-Shacoori Z, Ehrmann E, Dupont A, Barloy-Hubler F, Bousarghin L, et al. Oral Gram-negative anaerobic bacilli as a reservoir of $\beta$-lactam resistance genes facilitating infections with multiresistant bacteria. Int J Antimicrob Agents, 2015; 45(2): 99-105. doi: 10.1016/j.ijantimicag. 2014.10 .003

8. Brito-Aragão MG, Fernandes-Gomes FI, Ruliglesio-Rocha F, Teixeira-Pinto V. Prevalence and Susceptibility of Enterobacteriaceae Isolated from the Saliva of Students from the Northeast of Brazil. Glob J Med Res. 2016;16 (2):13-17. https:// globaljournals.org/GJMR_Volume16/3-Prevalence-and-Susceptibility.pdf

9. Leão-Vasconcelos LS, Lima AB, Costa Dde M, Rocha-Vilefort, LO, Oliveira AC, Gonçalve NF, et al. Enterobacteriaceae isolates from the oral cavity of workers in a Brazilian oncology hospital. Rev Inst Med Trop Sao Paulo. 2015;57(2):121-127. doi:10.1590/S0036-46652015000200004

10. Bryskier A. Viridans group streptococci: a reservoir of resistant bacteria in oral cavities. Clin Microbiol Infect. 2002;8:65---9. doi: https://doi.org/10.1046/ j.1198-743x.2001.00398.x

11. Handal T, Olsen I, Walker CB, Caugant DA. Beta-lactamase production and antimicrobial susceptibility of subgingival bacteria from refractory periodontitis. Oral Microbiol Immunol. 2004 ;19:303-8. doi: 10.1111/j.1399-302x.2004.00159.x

12. Sukumar S, Roberts AP, Martin FE, Adler CJ. Metagenomic insights into transferable antibiotic resistance in oral bacteria. J Dent Res 2016. https://doi. org/10.1177/0022034516648944

13. Brolund A, Sandegren L. Characterization of ESBL disseminating plasmids. Infect Dis. 2016; 48:18-25. doi: 10.3109/23744235.2015.1062536. Epub 2015 Jul 1 .

14. Sutcliffe J, Grebe T, Tait-Kamradt A, Wondrack Lillian. Detection of Erythromycin-Resistant Determinants by PCR. Antimicrob Agents Chemother. 1996; 40(11):2562-2566. PMID: 8913465; PMCID: PMC163576.
15. Villedieu A, Diaz-Torres ML, Roberts AP, Hunt N, McNab R,. Spratt DA, et al. Genetic Basis of Erythromycin Resistance in Oral Bacteria. Antimicrob Agents Chemoth. 2004; 48(6): 2298-2301. doi: 10.1128/AAC.48.6.2298-2301.2004.

16. Zhu L, Li Q, Li GC, Wu W, Pei N, He Y-x. Prevalence of erythromycin resistance genes among clinical isolates of viridians group Streptococci. Biomed Res. 2017; 28 (3): 1272-1275.

17. Clinical Laboratory Standards Institute. Performance standars for antimicrobial susceptibility testing, 27th informational supplement, 2017; M100-S27. Wayne, PA, USA.

18. Ying CM, Ling TK, Lee CC, Ling JM. Characterization of carbapenem-resistant Acinetobacter baumannii in Shanghai and Hong Kong. J. Med. Microbiol. 2006; 55:799-802. doi: 10.1099/jmm.0.46117-0

19. Mendes RE, Castanheira M, Garcia P, Guzman M, Toleman MA, Walsh TM, et al. First isolation of blaVIM-2 in Latin America: report from the SENTRY Antimicrobial Surveillance Program. Antimicrob Agents Chemother. 2004; 48:1433-34. doi: 10.1128/aac.48.4.1433-1434.2004

20. Mabilat C, Courvalin P. Development of oligotyping for characterization and molecular epidemiology of TEM $\beta$-lactamases in members of the family Enterobacteriaceae. Antimicrob Agents Chemother. 1990;34 (1): 2210-6. doi:10.1128/aac.34.11.2210

21. Herrera-León S, González-Sanz R, Rodríguez I, Rodicio MR, Echeita MA. Spread of a multiresistant CTX-M-9-producing Salmonella enterica serotype Virchow phage type 19 in Spain. Eur J Clin Microbiol Infect Dis. 2010;29:901-905. doi: 10.1007/ s10096-010-0939-6

22. Kuo H, Yang C, Lin M, Cheng W, Tiene N, Liou M. Distribution of blaOXA-carrying imipenem-resistant Acinetobacter spp. in 3 hospitals in Taiwan. Diag. Microbiol. Infect. Dis. 2010; 66: 195-199. doi: 10.1128/AAC.00779-13

23. Coudron PE, Moland ES, Thomson KS. Occurrence and detection of AmpC beta- lactamases among Es- 
cherichia coli, Klebsiella pneumoniae, and Proteus mirabilis isolates at a veterans medical center", J Clin Microbiol. 2000; 38(5):1791-6.

24. Magiorakos AP, Srinivasan A, Carey RB, Carmeli Y, Falagas ME, Giske CG, et al. Multidrug-resistant, extensively drug-resistant and pandrug-resistant bacteria: An international expert proposal for interim standard definitions for acquired resistance. Clin Microbiol Infec. 2012; 18(3):268-281. doi: 10.1111/j.1469-0691.2011.03570.x. Epub 2011 Jul 27.

25. Ferreira PVA, Amêndola I, Dias de Oliveira L, Gonçalves e Ssilva CR, VPL Mariella, Ferreira dos Santo SS. Prevalence and Sensitivity of BaciIli and Pseudomonas in the Newborn's Oral Cavity. Braz. Dent. J. 2017; 8 (4): 423-427. http://dx.doi. org/10.1590/0103-6440201601205

26. Zuanazzi D, Souto R, Mattos MB, Zuanazzi MR, Tura BR, Sansone C, et al. Prevalence of potential bacterial respiratory pathogens in the oral cavity of hospitalized individuals. Arch Oral Biol 2010;55(1):21-8.

27. Jones DJ, Munro CL. Oral care and the risk of bloodstream infections in mechanically ventilated adults: a review. Intensive Crit Care Nurs 2008;24(3):152-61.

28. Gaetti-Jardim EC, Marqueti AC, Faverani LP, Gaetti-Jardim Jr E. Antimicrobial resistance of aerobes and facultative anaerobes isolated from the oral cavity. J. Appl. Oral Sci. 2010; 18 ( 6 ): 551-559. doi: 10.1590/s1678-77572010000600004

29. Thomson KS. Extended-spectrum-lactamase, AmpC and carbapenemase issues. J Clin Microbiol. 2010;48:1019-1025. doi: 10.1128/JCM.00219-10. Epub 2010 Feb 24.

30. Coutinho V, Paiva RM, Reiter KC, de-Paris F, Barth AL, Machado AB, et al. Distribution of erm genes and low prevalence of inducible resistance to clindamycin among staphylococci isolates. Braz J Infect Dis. 2010; 14(6): 564-568. http://dx.doi. org/10.1590/S1413-86702010000600004
31. Goudarzi G, Tahmasbi F, Anbari K, Ghafarzadeh M. Distribution of Genes Encoding Resistance to Macrolides Among Staphylococci Isolated From the Nasal Cavity of Hospital Employees in Khorramabad, Iran, Iran Red Crescent Med J. 2016; 18(2):e25701. doi: 10.5812/ircmj.25701.

32. Aracil B, Minambres M, Oteo J, Torres C, Gomez-Garces JL, Alos JI. High prevalence of erythromycin-resistant and clindamycin-susceptible ( $M$ phenotype) viridans group streptococci from pharyngeal samples: a reservoir of mef genes in commensal bacteria. J. Antimicrob. Chemother. 2001;48:592-594. https://doi.org/10.1093/ jac/48.4.592

33. Luna VA, Heiken M, Judge K, Ulep C, Van Kirk N, Luis $\mathrm{H}$, et al. Distribution of $\operatorname{mef}(\mathrm{A})$ in gram-positive bacteria from healthy Portuguese children. Antimicrob. Agents Chemother. 2002; 46:25132517. 86702010000600004\&lng=en. http://dx.doi. org/10.1590/S1413-86702010000600004

34. Sutcliffe J, Tait-Kamradt A, Wondrack L. Streptococcus pneumonia and Streptococcus pyogenes resistant to macrolides but sensitive to clindamycin: a common resistance pattern mediated by efflux system. Antimicrob Agents Chemother. 1996;40:1817-24.

35. Liu N, Ando T, Ishiguro K, Maeda O, Watanabe, O, Funasaka K, et al. Characterization of bacterial biota in the distal esophagus of Japanese patients with reflux esophagitis and Barrett's esophagus. BMC Infect. Dis. 2013;13:130. doi:10.1186/1471-2334$13-130$

36. Mueller S, Saunier, K, Hanisch, C, Norin, E, Alm, L, Midtvedt, T, et al.. Differences in fecal microbiota in different European study populations in relation to age, gender, and country: a cross-sectional study. Appl. Environ. Microbiol. 2006; 72: 1027-1033. doi: 10.1128/AEM.72.2.1027-1033.

37. Gutierrez, D., \& Sánchez Mora, R. (2018). Tratamientos alternativos de medicina tradicional para Chlamydia trachomatis, agente causal de una infección asintomática. NOVA, 16(30), 65-74. Disponi- 
ble en: https://revistas.unicolmayor.edu.co/index. $\mathrm{php} /$ nova/article/view/869

38. Bejarano, O., Pérez, C., \& Mora, S. (2018). Resistencia microbiana desde una perspectiva metagenómica. NOVA, 16(29), 91-100. Disponible en: https://revistas.unicolmayor.edu.co/index.php/nova/ article/view/844 